\title{
ELABORAÇÃO DE BOLOS ENRIQUECIDOS COM SEMENTE DE ABÓBORA: AVALIAÇÃO QUÍMICA, FÍSICA E SENSORIAL
}

\author{
CAROLINE BITENCOURT * \\ FABIANA LEMOS GOULARTE DUTRA ** \\ VÂNIA ZANELLA PINTO ** \\ ELIZABETE HELBIG*** \\ LÚCIA ROTA BORGES ****
}

\begin{abstract}
Neste estudo avaliou-se o efeito da adição de farinha de semente de abóbora (FSA) nas propriedades químicas, físicas e sensoriais de bolos. A partir da formulação padrão foram elaborados três bolos contendo 7,5\%, $15 \%$ e $30 \%$ de FSA em substituição parcial à farinha de trigo. Os bolos com diferentes concentrações de FSA apresentaram maiores teores de fibras, proteínas e lipídeos em comparação ao bolo com farinha de trigo. Quanto ao conteúdo de tocoferois, os bolos com $15 \%$ e $30 \%$ de FSA apresentaram os maiores valores. A análise sensorial mostrou boa aceitabilidade dos bolos, apesar da formulação com $30 \%$ de FSA apresentar médias menores para os atributos cor e aparência.
\end{abstract}

* Graduada em Nutrição, Universidade Federal de Pelotas (UFPel), Pelotas, RS (e-mail: carol.r.bitencourt@ hotmail.com).

** Doutorandas em Ciência e Tecnologia de Alimentos, Departamento de Ciência e Tecnologia Agroindustrial, UFPEL, Pelotas, RS (e-mail: fgoularte@hotmail.com; vania_vzp@hotmail.com).

*** Doutora em Ciência e Tecnologia de Alimentos, Professora, Faculdade de Nutrição, Departamento de Ciência e Tecnologia Agroindustrial, UFPEL, Pelotas, RS (e-mail: helbignt@gmail.com).

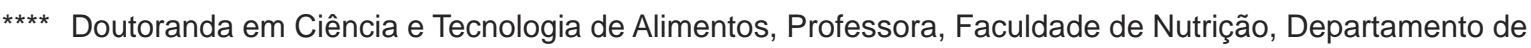
Ciência e Tecnologia Agroindustrial, UFPEL, Pelotas, RS (e-mail: luciarotaborges@yahoo.com.br). 


\section{INTRODUÇÃo}

As doenças crônicas não transmissíveis (DCNT), um dos maiores problemas de Saúde Pública, são responsáveis por aproximadamente $60 \%$ do total de mortes no mundo, segundo a Organização Mundial da Saúde (OMS). As modificações no padrão alimentar da população, evidenciadas pelo consumo de dietas com alta densidade energética, ricas em gordura (particularmente as de origem animal) e baixo teor de fibras, associadas ao sedentarismo, ao abuso de álcool e ao tabagismo, são considerados os principais fatores de risco para a ocorrência das DCNT (LENZ et al., 2009; GIMENO et al., 2011). Em função disso, a indústria alimentícia tem desenvolvido produtos enriquecidos que possam contribuir para a saúde dos consumidores, auxiliando na prevenção dessas doenças (MOURA et al., 2010).

A adição de farinhas mistas elaboradas a partir de vegetais, frutas e sementes à farinha de trigo vem sendo utilizada na indústria de panificação com a intenção de agregar valor nutricional a produtos como, pães, bolos e biscoitos (OLIVEIRA e REYES, 1990). Entre esses produtos, o bolo vem assumindo crescente importância no que se refere ao consumo e comercialização no Brasil (BORGES et al., 2006; CERQUEIRA et al., 2008; MOURA et al., 2010).

A adição de novos ingredientes exige o conhecimento das consequências desse incremento nas propriedades físico-químicas e sensoriais do produto elaborado em razão das modificações sensoriais, que podem diminuir sua aceitação pelo mercado consumidor (GIUNTINI, LAJOLO e MENEZES, 2003; COUTO, DERIVI e MENDEZ, 2004).

A farinha de semente de abóbora representa ingrediente alimentar com grande potencial de uso em função de suas características benéficas e relevantes à saúde (CERQUEIRA et al., 2008; PUMAR et al., 2008), sendo rica em fibras, proteínas, ácidos graxos poliinsaturados e sais minerais. Além disso, apresenta propriedades antioxidantes devido à presença de vitamina $E$, principalmente na forma dos isômeros $\gamma$-tocoferol e $\alpha$-tocoferol (GARCIA, KIMURA e MAURO, 2005). Apesar de sua importância nutricional é considerada resíduo agroindustrial, sendo desperdiçada em grande quantidade pela indústria processadora de vegetais (CERQUEIRA et al., 2008; MOURA et al., 2010).

O objetivo deste estudo foi avaliar o efeito da adição de diferentes concentrações de farinha de semente de abóbora nas propriedades químicas, físicas e sensoriais de bolos.

\section{MATERIAL E MÉTODOS}

O experimento foi executado no Laboratório de Técnica Dietética e no Laboratório de Análise de Alimentos da Faculdade de Nutrição, da Universidade Federal de Pelotas (UFPEL). O projeto foi aprovado pelo Comitê de Ética em Pesquisa da Faculdade de Medicina da UFPEL (Of. 29/11), sendo o Termo de Consentimento Livre e Esclarecido entregue aos julgadores no momento da análise sensorial.

\subsection{MATERIAL}

Foram utilizadas sementes de abóbora Kabutiá, provenientes do Restaurante Escola da UFPEL, sendo os demais ingredientes utilizados para a elaboração dos bolos adquiridos no comércio da cidade de Pelotas.

\subsubsection{Preparo da farinha de semente de abóbora}

Preparou-se a farinha de semente de abóbora (FSA) conforme metodologia descrita por Moura et al. (2010). As sementes foram lavadas para retirada do tecido vegetal, sanitizadas com solução de hipoclorito de sódio (200 ppm/15 minutos) e secas em estufas com circulação de ar a $40{ }^{\circ} \mathrm{C}$ por 18 horas. Após, as sementes foram trituradas em processador doméstico, torradas em 
fogo brando $\left(180^{\circ} \mathrm{C}\right)$ por 15 minutos, e resfriadas à temperatura ambiente. Peneirou-se a semente triturada em peneira de 40 mesh para obtenção da farinha.

\subsection{MÉTODOS}

\subsubsection{Elaboração dos bolos enriquecidos com FSA}

A partir da formulação padrão desenvolvida por Borges et al. (2006) foram elaborados bolos contendo $7,5 \%, 15 \%$ e $30 \%$ de FSA (Tabela 1).

TABELA 1 - INGREDIENTES UTILIZADOS NAS DIFERENTES FORMULAÇÕES

\begin{tabular}{lcccc}
\hline \multirow{2}{*}{ Ingredientes $(\mathbf{g})$} & \multicolumn{4}{c}{ Formulações* $^{*}$} \\
\cline { 2 - 5 } & $\mathbf{0}$ & $\mathbf{7 , 5} \%$ & $\mathbf{1 5 \%}$ & $\mathbf{3 0} \%$ \\
\hline Farinha de trigo & 100,0 & 92,5 & 85,0 & 70,0 \\
FSA & 0 & 7,5 & 15,0 & 30,0 \\
Açúcar & 76,7 & 76,7 & 76,7 & 76,7 \\
Ovo & 40,0 & 40,0 & 40,0 & 40,0 \\
Margarina & 20,0 & 20,0 & 20,0 & 20,0 \\
Fermento & 3,3 & 3,3 & 3,3 & 3,3 \\
Leite & 93,3 & 93,3 & 93,3 & 93,3 \\
\hline
\end{tabular}

* Porcentagem dos ingredientes em relação a $100 \%$ do peso total da farinha mista.

\subsubsection{Técnica de preparo dos bolos}

Os ovos, a margarina e o açúcar foram transferidos para batedeira doméstica e misturados por 1 minuto até formar creme homogêneo. Em seguida, adicionou-se a farinha de trigo refinada, a FSA (previamente homogeneizada) e o leite. O fermento químico em pó foi misturado manualmente. Distribuiu-se a massa em formas de alumínio $(6,0 \times 12,5 \times 4,5 \mathrm{~cm})$, previamente untadas com óleo de soja e forradas com papel manteiga, que foram levadas ao forno pré-aquecido por 15 minutos. $\mathrm{O}$ assamento durou 40 minutos a $185^{\circ} \mathrm{C}$, utilizando-se termômetro infra-vermelho TD-961 (ICEL Manaus) para o controle de temperatura (BORGES et al., 2006). Após assados e resfriados à temperatura ambiente, os bolos foram submetidos as análises.

\subsection{AVALIAÇÕES DOS BOLOS ENRIQUECIDOS COM FSA}

\subsubsection{Composição proximal}

Determinou-se a composição proximal dos bolos elaborados, segundo os procedimentos recomendados pela Association of Official Analytical Chemistry (AOAC, 1997). O conteúdo de cinzas (mufla $550^{\circ} \mathrm{C} / 6$ horas) e a umidade $\left(105^{\circ} \mathrm{C}\right.$ ) foram analisados por método gravimétrico e os teores de proteína bruta por meio da determinação de nitrogênio pelo método de Kjeldahl, usando-se o fator 6,25 para a conversão do nitrogênio total em proteína total (AOAC, 1997). Determinou-se o extrato etéreo em extrator intermitente de Soxhlet e o teor de fibras segundo Angelucci et al. (1987). 
Os carboidratos foram calculados por diferença dos demais componentes, usando-se a fórmula: 100 - (proteína bruta + lipídeos totais + fibra bruta + cinzas). Calculou-se o valor calórico dos bolos

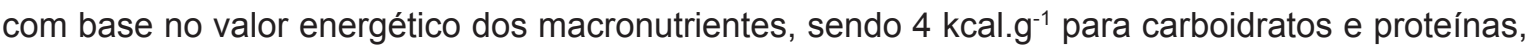
e 9 kcal.g-1 para lipídeos.

\subsubsection{Tocoferois}

A análise de tocoferois foi realizada de acordo com a metodologia descrita por Pestana et al. (2008) a partir do extrato etéreo. As amostras foram diluídas com solução de isopropanol:acetonitrila (7:3) e avaliadas em sistema de cromatografia a líquido de alta eficiência (HPLC), usandose cromatógrafo SHIMADZU, constituído por módulo de mistura dos solventes LC-10ATVP, desgaseificador FCV-10ALVP, bomba reodine DGU-14A, sistema de controle SCL-10AVP, forno da coluna CTO-10-ASVP e amostrador automático SIL-10AF. A separação ocorreu em coluna analítica de fase reversa, Synergi Fusion RP-80A, Phenomenex ${ }^{\circledR}(4,6 \mathrm{~cm} \times 150 \mathrm{~mm} \times 4 \mu \mathrm{m})$, tendo-se injetado $10 \mu \mathrm{L}$ de amostra. Utilizou-se detector de fluorescência com excitação de $290 \mathrm{~nm}$ e emissão a $330 \mathrm{~nm}$. Os dados foram adquiridos e processados com uso do software Class-VP.

Empregou-se como fase móvel inicial acetonitrila:metanol:isopropanol nas proporções 50:40:10 (v/v/v) por 10 minutos, alterada linearmente para acetonitrila:metanol:isopropanol (30:65:5 $\mathrm{v} / \mathrm{v} / \mathrm{v}$ ) até atingir 12 minutos, retornando-se linearmente para a fase móvel inicial até 15 minutos de análise. Utilizou-se fluxo constante de $1 \mathrm{~mL} \cdot \mathrm{min}^{-1}$. Para a identificação e quantificação dos tocoferois usou-se curva de calibração com os padrões de $\delta$-tocoferol e $\gamma$-tocoferol, ambos da Sigma Chemicals Co. (St. Louis, EUA), com pureza de $90 \%$ e $96 \%$, respectivamente, e a-tocoferol (pureza $99 \%$ ) adquirido da Merck (Darmstadt, Alemanha).

\subsubsection{Avaliação física}

As análises físicas dos bolos foram efetuadas de acordo com os procedimentos descritos pela American Association of Cereal Chemists (1995). Seis bolos de cada formulação, provenientes da mesma fornada e amostrados de forma aleatória, foram utilizados para a determinação dos parâmetros físicos de peso pré e pós-cocção, espessura antes e após o forneamento e o fator térmico de acordo com Araújo e Guerra (1995). Calculou-se o volume específico pela relação entre o volume aparente (determinado pelo método de deslocamento de sementes de painço) e o peso dos bolos assados, sendo expresso $\mathrm{em}^{\mathrm{cm}^{3}}$ (PIZZINATTO, MAGNO e CAMPAGNOLLI, 1993).

\subsubsection{Cor do miolo}

Determinou-se a cor do miolo dos bolos em Colorímetro Minolta (modelo CR-300) pelo sistema CIEL*a* $b^{*}$, no qual os valores de luminosidade $\left(L^{*}\right)$ variam entre zero (preto) e 100 (branco), os valores das coordenadas de cromaticidade $a^{*}$ e $b^{*}$ de $-a^{*}$ (verde) até $+a^{*}$ (vermelho), e de $-b^{*}$ (azul) até $+b^{*}$ (amarelo) (McGUIRE, 1992).

\subsection{PERFIL DE TEXTURA}

Determinou-se o perfil de textura dos bolos em Texturômetro (Texture Analyser TA.XTplus, Stable Micro Systems), sendo avaliados os parâmetros dureza, adesividade, flexibilidade, coesividade, gomosidade e mastigabilidade (ESTELLER e LANNES, 2005).

\subsection{AVALIAÇÃO SENSORIAL}

As amostras das quatro formulações foram avaliadas quanto aos atributos aparência, aroma, sabor, textura, cor e impressão global, mediante escala hedônica estruturada de nove pontos, 
com as extremidades ancoradas nos termos desgostei muitíssimo (1) e gostei muitíssimo (9).

O teste foi conduzido 24 horas após o forneamento em cabines individuais, iluminadas com lâmpadas fluorescentes. Contou-se com a participação de 30 julgadores não treinados, abordados aleatoriamente, entre professores, alunos e funcionários da Faculdade de Nutrição da UFPel, com idade entre 18 e 52 anos. Os julgadores receberam amostras com 8,0 $\pm 0,5 \mathrm{~g}$, simultaneamente, servidas e codificadas com números de três dígitos em blocos completos casualizados, conforme método desenvolvido por Minim (2006). Os julgadores foram orientados a ingerir água após cada degustação para limpeza do palato.

Para o cálculo do Índice de Aceitabilidade (IA) de cada preparação foi utilizada a seguinte expressão: IA $(\%)=A X 100 / B$, em que $A=$ nota média obtida para o produto e $B=$ nota máxima dada ao produto, sendo considerados aceitos os bolos que apresentaram IA igual ou superior a $70 \%$ (TEIXEIRA, MEINERT e BARBETTA, 1987).

\subsection{ANÁLISE ESTATISSTICA}

Os resultados das análises físicas, químicas e dos atributos sensoriais foram analisados com auxílio do software Statistica, versão 7.0 (STATSOFT, 2004). As variáveis foram apresentadas como médias e seus respectivos desvios padrões. Utilizou-se a análise de variância ANOVA e o teste de Tukey, considerando como nível de significância estatística o limite de $5 \%$.

\section{RESULTADOS E DISCUSSÃO}

\subsection{COMPOSIÇÃO PROXIMAL}

A composição proximal dos bolos elaborados está apresentada na Tabela 2.

Os bolos revelaram teor de umidade inferior a 9,14\%, porém sem diferença estatística entre os tratamentos. Em relação aos teores de lipídeos, fibras, proteínas e cinzas, os bolos com diferentes concentrações de FSA apresentaram valores maiores quando comparados ao bolo elaborado com farinha de trigo. O maior valor de proteínas deve-se ao alto conteúdo proteico encontrado na semente de abóbora, entre 25 a $31 \%$, podendo variar de acordo com a espécie (BORGES et al., 2006; CERQUEIRA et al., 2008).

Em relação à quantidade de lipídeos, os bolos com FSA apresentaram valores maiores quando comparados ao bolo com farinha de trigo. Esses achados podem estar relacionados à grande quantidade de ácidos graxos poliinsaturados ômega-6 (ácido linoleico) presente nessas sementes, os quais atuam na redução dos níveis de colesterol sanguíneo (ESUOSO et al., 1998).

$\mathrm{O}$ teor de cinzas foi maior nos bolos elaborados com FSA, devido à alta quantidade de minerais presente na semente (BORGES et al., 2006; CERQUEIRA et al., 2008). Os bolos elaborados com diferentes concentrações de FSA apresentaram diferença significativa entre si quanto ao conteúdo de proteínas, lipídeos e cinzas, cujos valores aumentaram conforme a elevação da concentração de FSA. Moura et al. (2010) ao analisarem a composição proximal de biscoitos tipo cookies, elaborados com semente de abóbora, encontraram valores semelhantes de proteínas e lipídeos aos verificados neste estudo.

A farinha de semente de abóbora apresentou 17,55 \% de fibra bruta, podendo ser considerada farinha com alto teor de fibras. De acordo com a Portaria $n^{\circ} 27$ do Ministério da Saúde, referente à informação nutricional, o produto com no mínimo $3 \%$ de fibras pode ser considerado como fonte de fibras e ao ultrapassar o mínimo de $6 \%$ será considerado com alto teor de fibras (BRASIL, 1998).

Pumar, Da Silva e Martins (2001), ao avaliarem frações obtidas no pré-preparo de abóbora bahiana, encontraram teores de fibra insolúvel de $29,49 \%$ em semente de abóbora integral, 24,88 \% na fração peneirada de semente de abóbora e 47,52 \% em semente de abóbora residual 
(fração retida na peneira).

Moscatto, Prudêncio-Ferreira e Haully (2004) analisaram bolos de chocolate enriquecidos com farinha de yacon e inulina (diferentes concentrações) em substuição à farinha de trigo. Observaram que a formulação contendo $40 \%$ de yacon e $6 \%$ de inulina apresentou $23,1 \%$ de fibra alimentar total, a formulação contendo $20 \%$ de farinha de yacon evidenciou $12 \%$ e a controle $9 \%$.

O valor calórico dos bolos elaborados com FSA foi maior que o dos produzidos com farinha de trigo. Isso provavelmente ocorreu pelo fato dos bolos com FSA apresentarem mais lipídeos. A formulação de bolos com maior valor calórico foi a com $30 \%$ de FSA, mostrando-se semelhante ao encontrado por Moura et al. (2010).

TABELA 2 - COMPOSIÇÃO PROXIMAL (\%) DOS BOLOS ELABORADOS

\begin{tabular}{ccccc}
\hline \multirow{2}{*}{ Componentes (\%) } & \multicolumn{4}{c}{ Formulações $^{*}$} \\
\cline { 2 - 5 } & $\mathbf{0}$ & $7,5 \%$ & $15 \%$ & $\mathbf{3 0 \%}$ \\
\hline Umidade & $8,47 \pm 0,35^{\mathrm{a}}$ & $8,50 \pm 0,42^{\mathrm{a}}$ & $8,57 \pm 0,47^{\mathrm{a}}$ & $9,14 \pm 0,14^{\mathrm{a}}$ \\
Cinzas & $1,06 \pm 0,02^{\mathrm{d}}$ & $1,14 \pm 0,02^{\mathrm{c}, \mathrm{d}}$ & $1,23 \pm 0,04^{\mathrm{b}}$ & $1,49 \pm 0,04^{\mathrm{a}}$ \\
Lipídeos & $6,61 \pm 0,50^{\mathrm{c}}$ & $8,65 \pm 0,27^{\mathrm{b}, \mathrm{c}}$ & $10,07 \pm 0,46^{\mathrm{b}}$ & $17,36 \pm 0,61^{\mathrm{a}}$ \\
Proteínas & $6,40 \pm 0,09^{\mathrm{d}}$ & $6,90 \pm 0,09^{\mathrm{c}}$ & $7,21 \pm 0,11^{\mathrm{b}}$ & $8,14 \pm 0,07^{\mathrm{a}}$ \\
Fibras & $0,26 \pm 0,03^{\mathrm{d}}$ & $0,76 \pm 0,02^{\mathrm{c}}$ & $1,34 \pm 0,10^{\mathrm{b}}$ & $2,40 \pm 0,19^{\mathrm{a}}$ \\
Carboidratos & $77,20 \pm 0,66^{\mathrm{a}}$ & $74,05 \pm 0,67^{\mathrm{b}}$ & $71,59 \pm 0,14^{\mathrm{c}}$ & $61,47 \pm 0,59^{\mathrm{d}}$ \\
Valor calórico (Kcal) & $393,92 \pm 2,14^{\mathrm{c}}$ & $401,68 \pm 1,52^{\mathrm{b}}$ & $405,80 \pm 4,21^{\mathrm{b}}$ & $434,65 \pm 3,05^{\mathrm{a}}$ \\
\hline
\end{tabular}

* 0 = bolo com farinha de trigo; $7,5 \%$ = bolo com $7,5 \%$ de FSA; $15 \%$ = bolo com $15 \%$ de FSA; $30 \%$ = bolo com $30 \%$ de FSA. "Carboidratos calculados por diferença em relação aos demais componentes. Resultados são médias de três determinações \pm desvio padrão. Letras diferentes nas linhas diferem significativamente pelo teste de Tukey $(p<0,05)$.

\subsection{TOCOFEROIS}

A Tabela 3 apresenta o conteúdo de tocoferois dos bolos enriquecidos com FSA.

TABELA 3 - CONTEÚDO DE TOCOFEROIS DOS BOLOS ELABORADOS *

\begin{tabular}{lcccc}
\hline \multirow{2}{*}{$\begin{array}{l}\text { Tocoferol } \\
(\mathbf{m g} / \mathbf{1 0 0} \mathrm{g})\end{array}$} & $\mathbf{0}$ & $\mathbf{7 , 5} \%$ & $\mathbf{1 5 \%}$ & $\mathbf{3 0 \%}$ \\
\cline { 2 - 5 } & $0,50 \pm 0,02^{\mathrm{c}}$ & $0,68 \pm 0,01^{\mathrm{c}}$ & $0,71 \pm 0,03^{\mathrm{a}, \mathrm{b}}$ & $0,78 \pm 0,03^{\mathrm{a}}$ \\
\hline $\mathrm{n}$ - tocoferol & $2,56 \pm 0,04^{\mathrm{b}}$ & $3,09 \pm 0,14^{\mathrm{b}}$ & $3,85 \pm 0,28^{\mathrm{a}}$ & $4,03 \pm 0,08^{\mathrm{a}}$ \\
\hline - tocoferol & 2
\end{tabular}

"Resultados são médias de três determinações \pm desvio padrão. Letras diferentes nas linhas diferem significativamente pelo teste de Tukey $(p<0,05)$." 0 = bolo com farinha de trigo; $7,5 \%=$ bolo com $7,5 \%$ de FSA; $15 \%$ = bolo com $15 \%$ de FSA; $30 \%=$ bolo com $30 \%$ de FSA. 
O bolo controle não diferiu significativamente da formulação com $7,5 \%$ de FSA quanto ao teor de $\mathrm{y}$-tocoferol e apresentou menor conteúdo que os bolos com $15 \%$ e $30 \%$ de FSA. Para o a-tocoferol, o tratamento com $30 \%$ não diferiu do bolo elaborado com $15 \%$ de FSA, sendo superior aos demais.

O gama-tocoferol constitui a forma predominante de tocoferol em sementes de gergelim, colza e linhaça (LECHNER, REITER e LORBEER, 1999; YAMASHITA, IKEDA e OBAYASHI, 2003).

\subsection{AVALIAÇÃO FÍSICA DOS BOLOS}

Na Tabela 4 encontram-se os dados referentes à perda de peso após o forneamento, fator térmico, aumento de espessura e volume específico das quatro formulações de bolo.

\section{TABELA 4 - PARÂMETROS FÍSICOS DOS BOLOS ELABORADOS *}

\begin{tabular}{lcccc}
\hline \multirow{2}{*}{ Parâmetros físicos } & \multicolumn{4}{c}{ Formulações ** } \\
\cline { 2 - 5 } & $\mathbf{0}$ & $\mathbf{7 , 5} \%$ & $\mathbf{1 5 \%}$ & $\mathbf{3 0 \%}$ \\
\hline $\begin{array}{l}\text { Perda de peso ao } \\
\text { assar (g) }\end{array}$ & $19,44 \pm 0,59^{\mathrm{a}}$ & $18,53 \pm 1,08^{\mathrm{a}, \mathrm{b}}$ & $18,63 \pm 0,90^{\mathrm{a}, \mathrm{b}}$ & $17,80 \pm 0,83^{\mathrm{b}}$ \\
Fator térmico & $0,80 \pm 0,01^{\mathrm{b}}$ & $0,81 \pm 0,01^{\mathrm{a}}$ & $0,81 \pm 0,01^{\mathrm{a}, \mathrm{b}}$ & $0,82 \pm 0,01^{\mathrm{a}}$ \\
$\begin{array}{l}\text { Aumento espessura } \\
(\mathrm{cm})\end{array}$ & $1,54 \pm 0,16^{\mathrm{a}}$ & $1,78 \pm 0,09^{\mathrm{a}}$ & $1,62 \pm 0,17^{\mathrm{a}}$ & $1,79 \pm 0,07^{\mathrm{a}}$ \\
$\begin{array}{l}\text { Volume específico } \\
(\mathrm{cm})\end{array}$ & $\left.2,48 \pm 0,1^{\mathrm{a}}\right)$ & $2,26 \pm 0,09^{\mathrm{b}}$ & $2,36 \pm 0,09^{\mathrm{a}, \mathrm{b}}$ & $2,35 \pm 0,13^{\mathrm{a}, \mathrm{b}}$ \\
\hline
\end{tabular}

"Resultados são médias de três determinações \pm desvio padrão. Letras diferentes nas linhas diferem significativamente pelo teste de Tukey $(p<0,05)$. ${ }^{*} 0=$ bolo com farinha de trigo; $7,5 \%=$ bolo com $7,5 \%$ de FSA; $15 \%=$ bolo com $15 \%$ de FSA; $30 \%$ = bolo com $30 \%$ de FSA.

A perda de peso ao assar demonstra a capacidade da massa em reter água, enquanto o volume específico torna-se importante para verificar a capacidade da farinha em se expandir e reter o gás no interior da massa durante o forneamento (OLIVEIRA e REYES, 1990; ESTELLER e LANNES, 2005). Observou-se perda de peso ao assar de 19,44 g, 18,53 g, 18,63 g e 17,80 g, respectivamente, para os bolos controle, $7,5 \%, 15 \%$ e $30 \%$ de FSA. Após o forneamento, o bolo com $30 \%$ de FSA apresentou a menor perda de peso, diferindo significativamente do controle (farinha de trigo).

Os bolos elaborados com farinha de trigo $(0,80)$ diferiram estatisticamente quanto ao fator térmico apenas dos bolos com $30 \%$ de FSA $(0,82)$. Menor valor de fator térmico para os bolos com farinha de trigo quando comparado aos com $30 \%$ de FSA demonstra menor perda de umidade durante a cocção. Esses efeitos podem estar relacionados ao fato das fibras promoverem retenção de água, gerando maior peso (OLIVEIRA e REYES, 1990).

De acordo com Saura-Calixto (1993), a capacidade de absorção de água é a propriedade física mais importante para as fibras alimentares, pois maior retenção de água favorece o uso das fibras como ingrediente em alimentos enriquecidos.

Oliveira e Reyes (1990) e Souza et al. (2000) constataram elevação na umidade de biscoitos conforme aumento no teor de fibras, indicando maior retenção de água em virtude das características hidrofílicas da fibra. Perez e Germani (2004) avaliaram biscoitos com farinha de berinjela e verificaram que quanto maior a quantidade de farinha de berinjela menor o peso do 
biscoito. Moura et al. (2010) analisaram biscoitos formulados com semente de abóbora e observaram menor peso do que os biscoitos com farinha de trigo antes e após o forneamento. Em relação ao aumento da espessura, não houve diferença estatística entre os tratamentos antes e após o forneamento.

Os bolos preparados com farinha de trigo apresentaram maiores volumes específicos. Analisando somente os bolos preparados com FSA observou-se que aumento na concentração de FSA eleva os valores de volume específico.

Vários fatores afetam o volume específico de bolos, como a qualidade dos ingredientes usados na formulação da massa, especialmente a farinha e os tratamentos usados durante o processamento. Estudo realizado por Moscatto, Prudêncio-Ferreira e Haully (2004) mostrou que os volumes específicos dos bolos formulados com a incorporação da farinha de yacon e inulina não diferiram significativamente entre si, assim como os bolos contendo $15 \%$ e $30 \%$ de FSA no presente estudo.

Na Tabela 5 estão apresentados os parâmetros de cor dos bolos, representados pelos valores de $L^{*}$ (luminosidade), $a^{*}$ e $b^{*}$ (cromaticidade).

A cor dos alimentos, atributo que pode influenciar a decisão de compra dos consumidores, representa indicador de pigmentos durante o escurecimento não enzimático e o processo de caramelização (IBANOGLU, 2002).

Todos os bolos elaborados com farinha de semente de abóbora apresentaram valores de $L^{*}$ e $b^{*}$ menores que aqueles com farinha de trigo, ou seja, coloração mais escura. Os bolos contendo $30 \%$ de FSA apresentaram os menores valores de $L^{*} e b^{*}$.

A cromaticidade ${ }^{*}$ diminuiu com o aumento da concentração de FSA. Collins e Falasinnu (1977) constataram que biscoitos elaborados com farelo de trigo e celulose, em diferentes concentrações, ficaram mais escuros que o biscoito controle. Resultados semelhantes foram obtidos por Leelavathi e Rao (1993) utilizando fibra de milho e Silva (1997) estudando farinha de jatobá.

A coloração dos bolos está relacionada principalmente com os ingredientes da formulação, sendo a farinha de semente de abóbora mais escura do que a farinha de trigo. Assim, maior teor dessa farinha na formulação dos bolos provoca escurecimento.

Perez e Germani (2007) avaliaram biscoitos com farinha de beringela e verificaram que os biscoitos com maior teor de fibras apresentaram coloração mais escura.

TABELA 5 - PARÂMETROS DE COR DOS BOLOS ELABORADOS *

\begin{tabular}{cccc}
\hline Formulações $^{* *}$ & $\mathbf{L}^{*}$ & $\mathbf{a}^{\mathbf{*}}$ & $\mathbf{b}^{*}$ \\
\hline 0 & $75,94^{\mathrm{a}}$ & $0,61^{\mathrm{a}}$ & $21,82^{\mathrm{c}}$ \\
$7,5 \%$ & $67,33^{\mathrm{b}}$ & $-2,23^{\mathrm{b}}$ & $27,83^{\mathrm{b}}$ \\
$15 \%$ & $58,97^{\mathrm{c}}$ & $-4,06^{\mathrm{c}}$ & $29,17^{\mathrm{b}}$ \\
$30 \%$ & $51,97^{\mathrm{a}}$ & $-6,43^{\mathrm{d}}$ & $31,18^{\mathrm{a}}$ \\
\hline
\end{tabular}

"Resultados são médias de três determinações. Letras diferentes nas linhas diferem significativamente pelo teste de Tukey $(p<0,05)$. " $0=$ bolo com farinha de trigo; $7,5 \%=$ bolo com $7,5 \%$ de FSA; $15 \%=$ bolo com $15 \%$ de FSA; $30 \%=$ bolo com $30 \%$ de FSA.

\subsection{PERFIL DE TEXTURA}

A textura indica de forma direta a qualidade dos produtos de panificação. Na Tabela 6 são apresentados os resultados do perfil de textura dos bolos elaborados com diferentes concentrações de FSA.

Define-se a textura de alimentos como um grupo de características físicas provenientes 
dos elementos estruturais do produto (SZCZESNIAK, 2002). Essas características são quantificadas mediante análises que podem ser sensoriais ou instrumentais (ROSENTHAL, 1999).

TABELA 6 - PERFIL DE TEXTURA DOS BOLOS ELABORADOS *

\begin{tabular}{lcccc}
\hline Parâmetros & \multicolumn{4}{c}{ Formulações ** } \\
\cline { 2 - 5 } & $\mathbf{0}$ & $\mathbf{7 , 5} \%$ & $\mathbf{1 5 \%}$ & $\mathbf{3 0} \%$ \\
\hline Dureza $(\mathrm{N})$ & $3,59 \pm 0,02^{\mathrm{b}}$ & $3,23 \pm 0,01^{\mathrm{b}}$ & $3,09 \pm 0,10^{\mathrm{b}}$ & $4,49 \pm 0,20^{\mathrm{a}}$ \\
Adesividade & $-0,96 \pm 0,20^{\mathrm{a}}$ & $-1,44 \pm 0,20^{\mathrm{a}}$ & $-1,95 \pm 0,10^{\mathrm{a}, \mathrm{b}}$ & $-3,12 \pm 0,30^{\mathrm{b}}$ \\
Coesividade & $0,55 \pm 0,02^{\mathrm{a}}$ & $0,49 \pm 0,01^{\mathrm{b}}$ & $0,48 \pm 0,01^{\mathrm{b}}$ & $0,49 \pm 0,01^{\mathrm{b}}$ \\
Mastigabilidade & $1,73 \pm 0,05^{\mathrm{a}, \mathrm{b}}$ & $1,29 \pm 0,10^{\mathrm{b}, \mathrm{c}}$ & $1,22 \pm 0,02^{\mathrm{c}}$ & $1,92 \pm 0,01^{\mathrm{a}}$ \\
\hline
\end{tabular}

"Resultados são médias de três determinações \pm desvio padrão. Letras diferentes nas linhas diferem significativamente pelo teste de Tukey $(p<0,05)$. " 0 = bolo com farinha de trigo; $7,5 \%=$ bolo com $7,5 \%$ de FSA; $15 \%=$ bolo com $15 \%$ de FSA; $30 \%=$ bolo com $30 \%$ de FSA.

Apenas o bolo com $30 \%$ de FSA apresentou maior dureza (Tabela 6), provavelmente como resultado da presença de proteínas e fibras na FSA (BERNARDINO, 2011). Arora e Camire (1994) verificaram que a substituição de parte de farinha de trigo por fibras (frutas, açúcar da beterraba, farelo de trigo pela celulose ou casca de batata) gerou bolos e biscoitos com maior firmeza e conservação da textura durante a estocagem. Em cookies, a substituição de farinha por farelo de trigo e celulose resultou em texturas mais firmes.

A adesividade apresentou decréscimo com o aumento da concentração de FSA. O aumento na umidade da massa, a presença de sacarose e ingredientes com propriedades umectantes (como fibras) podem influenciar a interface amostra/probe no texturômetro (ESTELLER e LANNES, 2005).

As amostras contendo FSA ficaram menos coesas do que o controle (Tabela 6), indicando que a incorporação de FSA torna os bolos ligeiramente mais propensos à desintegração e que é possível produzir bolo rico em fibras com a mesma coesão (BERNARDINO, 2011). Os resultados obtidos para o parâmetro coesividade foram semelhantes, exceto o controle, aos relatados por Grigelmo-Miguel, Carrerasboladeras e Martin-Belloso (2001), os quais variaram de 0,45 a 0,5 para muffins elaborados com diferentes concentrações de fibra de pêssego. No entanto, são inferiores aos obtidos por Gómez et al. (2010), que mostraram variação de 0,70 a 0,58 para bolos elaborados com farelo de trigo, farelo de aveia e celulose microcristalina em diferentes concentrações.

A mastigabilidade caracteriza-se pela energia requerida para mastigar 0 alimento até a deglutição. Pode-se verificar que a amostra com $30 \%$ de FSA apresentou maior valor para mastigabilidade, comportamento semelhante à dureza (Tabela 6). Segundo Grigelmo-Miguel, Carrerasboladeras e Martin-Belloso (2001), a substituição de gordura por fibras em muffins aumentou a sua densidade e reduziu o número de bolhas de ar, elevando a força necessária para a compressão.

\subsection{AVALIAÇÃO SENSORIAL}

Participaram do teste de aceitação 30 julgadores não treinados, com média de idade de 24,3 anos, sendo $86,6 \%$ do sexo feminino. Em relação ao hábito de consumo de bolos, 56,7 \% dos julgadores assinalaram a alternativa eventualmente; $26,7 \%$ uma vez por semana; $13,3 \%$ duas vezes por semana e apenas 3,3 \% não consomem bolos.

A Tabela 7 apresenta o índice de aceitabilidade (IA) das formulações quanto aos atributos 
aparência, aroma, sabor, textura, impressão global e cor. A análise sensorial demonstrou que os bolos apresentaram IA superior a $70 \%$, com exceção do bolo elaborado com $30 \%$ de FSA para os atributos aparência e cor e do bolo com $15 \%$ de FSA para o atributo cor.

\section{TABELA 7 - ÍNDICE DE ACEITABILIDADE DAS FORMULAÇÕES* ELABORADAS QUANTO AOS ATRIBUTOS APARÊNCIA, AROMA, SABOR, TEXTURA, IMPRESSÃO GLOBAL E COR}

\begin{tabular}{lcccc}
\hline \multirow{2}{*}{ Atributos } & \multicolumn{4}{c}{ Índice de Aceitabilidade (\%) dos bolos ** } \\
\cline { 2 - 5 } & $\mathbf{0}$ & $\mathbf{7 , 5} \%$ & $\mathbf{1 5 \%}$ & $\mathbf{3 0} \%$ \\
\hline Aparência & 90,70 & 83,30 & 71,90 & 65,20 \\
Aroma & 87,80 & 84,10 & 79,60 & 75,90 \\
Sabor & 88,90 & 84,80 & 77,80 & 72,20 \\
Textura & 91,10 & 83,00 & 87,80 & 76,30 \\
Impressão global & 88,90 & 84,40 & 77,80 & 70,00 \\
Cor & 89,60 & 81,10 & 69,30 & 57,40 \\
\hline
\end{tabular}

${ }^{*} 0=$ bolo com farinha de trigo; $7,5 \%=$ bolo com $7,5 \%$ de FSA; $15 \%=$ bolo com $15 \%$ de FSA; $30 \%=$ bolo com $30 \%$ de FSA. **Índice de boa aceitação $\geq 70 \%$.

Para Teixeira, Meinert e Barbetta (1987), o produto pode ser considerado aceito em termos de suas propriedades sensoriais quando alcançar IA de no mínimo $70 \%$.

A Tabela 8 apresenta as médias atribuídas pelos julgadores aos bolos avaliados.

TABELA 8 - RESULTADOS DA ANÁLISE SENSORIAL PARA AVALIAÇÃO DAS AMOSTRAS DE BOLO, CONSIDERANDO OS ATRIBUTOS APARÊNCIA, AROMA, SABOR, TEXTURA, IMPRESSÃO GLOBAL E COR *

\begin{tabular}{lcccc}
\hline \multirow{2}{*}{ Atributos } & \multicolumn{4}{c}{ Formulações $^{\star *}$} \\
\cline { 2 - 5 } & $\mathbf{0}$ & $\mathbf{7 , 5} \%$ & $\mathbf{1 5 \%}$ & $\mathbf{3 0 \%}$ \\
\hline Aparência & $8,16 \pm 1,4^{\mathrm{a}}$ & $7,50 \pm 1,5^{\mathrm{a}, \mathrm{b}}$ & $6,46 \pm 1,6^{\mathrm{b}, \mathrm{c}}$ & $5,86 \pm 2,1^{\mathrm{c}}$ \\
Aroma & $7,90 \pm 1,2^{\mathrm{a}}$ & $7,56 \pm 1,5^{\mathrm{a}}$ & $7,16 \pm 1,8^{\mathrm{a}}$ & $6,83 \pm 2,0^{\mathrm{a}}$ \\
Sabor & $8,00 \pm 1,2^{\mathrm{a}}$ & $7,63 \pm 1,6^{\mathrm{a}, \mathrm{b}}$ & $7,00 \pm 1,8^{\mathrm{a}, \mathrm{b}}$ & $6,50 \pm 2,2^{\mathrm{b}}$ \\
Textura & $8,20 \pm 1,0^{\mathrm{a}}$ & $7,46 \pm 1,8^{\mathrm{a}, \mathrm{b}}$ & $7,90 \pm 1,2^{\mathrm{a}}$ & $6,86 \pm 2,1^{\mathrm{b}}$ \\
Impressão global & $8,00 \pm 1,1^{\mathrm{a}}$ & $7,60 \pm 1,4^{\mathrm{a}}$ & $7,00 \pm 1,6^{\mathrm{a}, \mathrm{b}}$ & $6,30 \pm 2,0^{\mathrm{b}}$ \\
Cor & $8,06 \pm 1,5^{\mathrm{a}}$ & $7,30 \pm 1,9^{\mathrm{a}, \mathrm{c}}$ & $6,23 \pm 2,0^{\mathrm{b}, \mathrm{c}}$ & $5,16 \pm 2,2^{\mathrm{b}}$ \\
\hline
\end{tabular}

'Resultados são médias de três determinações \pm desvio padrão. Letras diferentes nas linhas diferem significativamente pelo teste de Tukey $(p<0,05)$. " 0 = bolo com farinha de trigo; $7,5 \%=$ bolo com $7,5 \%$ de FSA; $15 \%=$ bolo com $15 \%$ de FSA; $30 \%=$ bolo com $30 \%$ de FSA. 
Em relação ao atributo aparência, o bolo elaborado com farinha de trigo apresentou a maior nota, diferindo significativamente do bolo com $30 \%$ de FSA (menor média). Quanto ao aroma, verificou-se comportamento semelhante para aceitação sensorial desse atributo em todas as amostras.

Os bolos elaborados com farinha de trigo e $7,5 \%$ de FSA foram os mais aceitos entre os avaliadores e não diferiram entre si em relação a todos os atributos. As formulações contendo $7,5 \%$ e $15 \%$ de FSA e sem FSA não diferiram estatisticamente em termos de impressão global e textura.

O índice de aceitação dos bolos diminui com o aumento da concentração de FSA em substituição à farinha de trigo. Borges et al. (2006) avaliaram sensorialmente bolos com diferentes percentuais de farinha de aveia acrescida à farinha de trigo. Obtiveram melhores índices de aceitabilidade para formulações que não continham a farinha de aveia e a contendo $30 \%$ dessa farinha.

Silveira et al. (2008) obtiveram $60 \%$ de aprovação em teste de aceitabilidade de mini panetone integral enriquecido com farinha de trigo integral. Os julgadores que não gostaram da textura do produto afirmaram que o panetone apresentou-se pesado.

Aguilar, Palomo e Bressani (2004) realizaram análise sensorial, por meio de teste afetivo, de pão formulado com $30 \%$ de farinha de arroz em substituição parcial a farinha de trigo. Constataram bom índice de aceitabilidade e melhor qualidade nutricional do produto.

Dotto (2004) usou teste sensorial afetivo para avaliar formulações de bolos enriquecidos com farinha de banana verde (FBV). O bolo mais aceito continha $30 \%$ dessa farinha, indicando que a coloração escura conferida à massa pela FBV mostrou-se atrativa para o consumidor. Entretanto, quando Fasolin et al. (2007) incorporaram FBV em biscoitos cookies houve redução na aceitação do produto conforme aumento do percentual de FBV. No presente estudo, o aumento do teor de FSA nas formulações provocou diminuição das médias para o atributo cor.

Segundo Dutcosky (1996) as pessoas tendem a aceitar melhor os alimentos preparados a partir de ingredientes tradicionalmente estabelecidos e próximos aos seus hábitos alimentares. A preferência dos indivíduos por determinados alimentos resulta do relacionamento sinérgico entre fatores ambientais, biológicos, ecológicos e sócio-culturais.

\section{CONCLUSÃO}

O uso da farinha de semente de abóbora em substituição parcial à farinha de trigo melhorou a qualidade nutricional dos bolos, evidenciada pelo aumento nos teores de fibras, proteínas, minerais e lipídeos. Quanto ao conteúdo de tocoferois, tanto o bolo com $15 \%$ quanto o bolo com $30 \%$ de FSA apresentaram os maiores valores. Além disso, as diferentes formulações obtiveram bons índices de aceitabilidade pelos julgadores.

\section{ABSTRACT \\ ELABORATION OF CAKES ENRICHED BY PUMPKIN SEEDS: CHEMICAL, PHYSICAL AND SENSORY ASSESSMENT}

This study has evaluated the effect of the addition of pumpkin seed meal (FSA) in the chemical, physical and sensory characteristics of cakes. Three cakes were prepared from a standard formulation containing $7.5 \%$, $15 \%$ and $30 \%$ of FSA as a partial substitute for wheat flour. The cakes with different concentrations of FSA had higher levels of fiber, protein and lipids in comparison with the wheat flour cake. Higher values of tocopherols were observed in the cakes with 15 and $30 \%$ of FSA. Despite the formulation with $30 \%$ FSA obtaining the lower averages for color and appearance attributes, the sensory analysis has indicated good acceptability of the cakes.

KEY-WORDS: PUMPKIN SEEDS; CAKES; SENSORY ANALYSIS. 


\section{REFERÊNCIAS}

1 AGUILAR, M.J.R.; PALOMO, P.; BRESSANI, R. Desarrollo de un producto de panificación apto para el adulto mayor a base de harina de trigo y harina de arroz. Archivos Latinoamericano de Nutrición, v.54, n.3, p.314-321, 2004.

2 American Association of Cereal Chemists (AACC). Approved methods of the American Association of Cereal Chemists. $9^{\text {th }}$ ed. Saint Paul, 1995. v. 2.

3 ANGELUCCI, E.; CARVALHO, C.R.L.; CARVALHO, P.R.N.; FIGUEIREDO, I.B.; MANTOVANI, D.M.B.; MORAES, R. M. Manual técnico de análises de alimentos. Campinas: Instituto de Tecnologia de Alimentos, 1987. p. 52-53.

4 Association of Official Analytical Chemists (AOAC). Official methods of the AOAC International. $16^{\text {th }}$ ed. Arlington, 1997. $850 \mathrm{p}$.

5 ARORA, A.; CAMIRE, M.E. Performance of potato peels in muffins and cookies. Food Research International, v.27, p.15-22, 1994.

6 ARAÚJO, M.O.D.; GUERRA, T.M.M. Alimentos "per capita". 2. ed. Natal: Editora Universitária - UFRN, 1995. 272 p.

7 BERNARDINO, M.A. Caracterização e aplicação da farinha do bagaço da cana-de-açúcar em bolo. $2011.51 \mathrm{f}$. Dissertação (Mestrado em Engenharia de Alimentos), Departamento de Engenharia de Alimentos, Universidade de São Paulo, Pirassununga, 2011.

8 BORGES, J.T.S. et al. Utilização de farinha mista de aveia e trigo na elaboração de bolos. Boletim do CEPPA, v.24, n.1, p.145-162, 2006

9 BRASIL. Ministério da Saúde. Portaria n. 27 de 13 de janeiro de 1998. Regulamento técnico referente à informação nutricional complementar. Diário Oficial [da] República Federativa do Brasil, Brasília, n. 11, jul. 1998. Seção 1, p. 4-13.

10 CERQUEIRA, P. M. et al. Efeito da farinha de semente de abóbora (Curcubita maxima, L.) sobre o metabolismo glicídico e lipídico em ratos. Revista de Nutrição, v.21, n.2, p.129-136, 2008.

11 COLLINS, J.L.; FALASINNU, G.A. Dietary fiber as an ingredient in cookies. Tennessee Farm and Home Science, v.101, p.21-24, Jan.1977.

12 COUTO, S.R.M.; DERIVI, S.C.N.; MENDEZ, M.H.M. Utilização tecnológica de subprodutos da indústria de vegetais. Higiene Alimentar, v.18, n.124, p.12-22, 2004.

13 DOTTO, D.C. Obtenção de farinha de banana verde, sua caracterização quanto a alguns componentes e avaliação de seu uso em formulações de bolo como substituta parcial da farinha de trigo. 2004. $51 \mathrm{f}$. Monografia (Especialista em Engenharia Química), Departamento de Engenharia Química, Universidade Estadual do Oeste do Paraná, Toledo, 2004.

14 DUTCOSKY, S.D. Análise sensorial de alimentos. Curitiba: Editora Universitária Champagnat, 1996.

15 ESTELLER, M.S.; LANNES, S.C.S. Parâmetros complementares para fixação de identidade e qualidade de produtos panificados. Ciência e Tecnologia de Alimentos, Campinas, v.25, n.4, p. 802-806, 2005.

16 ESUOSO, K. et al. Chemical composition and potential of some underutilized tropical biomass. I. Fluted pumpkin (Telfairia occidentalis). Food Chemistry, v.61, n.4, p.487-92, 1998.

17 FASOLIN, L.H. et al. Biscoitos produzidos com farinha: avaliações química, física e sensorial. Ciência e Tecnologia de Alimentos, v.27, n. 3, p.524-529, 2007.

18 GARCIA, C.C.; KIMURA, M.; MAURO, M.A. Efeito da temperatura de secagem na retenção de carotenóides de abóbora (Cucúrbita moschata). In: SIMPÓSIO LATINO-AMERICANO DE CIÊNCIA DE ALIMENTOS, 6., 2005, Campinas. Anais... Campinas: Unicamp, 2005. p. 1-1.

19 GIMENO, S.G.A. et al. Padrões de consumo de alimentos e fatores associados em adultos de Ribeirão Preto, São Paulo, Brasil: projeto OBEDIARP. Caderno de Saúde Pública, v.27, p.533-545, 2011.

20 GIUNTINI, E.B.; LAJOLO, F.M.; MENEZES, E.W. Potencial de fibra alimentar em países ibero-americanos: alimentos, produtos e resíduos. Archivos Latinoamericanos de Nutrición, v.53, n.1, p.14-20, 2003.

21 GÓMEZ, M.; MORALEJA, A.; OLIETE, B.; RUIZ, E.; CABALLERO, P.A. Effect of fibre size on the quality of fibre-enriched layer cakes. LWT - Food Science and Technology, v.43, n.1, p.33-38, 2010.

22 GRIGELMO-MIGUEL, N.; CARRERASBOLADERAS, E.; MARTIN-BELLOSO, O. Influence of the addition of peach dietary fiber in composition, physical properties and acceptability of reduced-fat muffins. Food Science and Technology, v.7, n.5, p.425-431, 2001. 
23 IBANOGLU, E. Kinetic study in colour changes in wheat germ due to heat. Journal of Food Engineering, v.51 p.209213, 2002.

24 LECHNER, M.; REITER, B.; LORBEER, E. Determination of tocopherols as sterols in vegetable oils by solid-phase extraction and subsequent capillary gas chromatographic analysis. Journal of Chromatography Analitycal, v.857, p.231-8, 1999.

25 LEELAVATHI, K.; RAO, H.P. Development of high fiber biscuits using wheat bran. Journal of Food Science Technology, v.30, n.3, p.187-191, 1993.

26 LENZ, A. et al. Socioeconomic, demographic and lifestyle factors associated with dietary patterns of women living in Southern Brazil. Caderno de Saúde Pública, v.25, p.1297-306, 2009.

27 McGUIRE, R. G. Reporting of objective color measurements. Horticultural Science, v. 27, n. 12, p. 1254-1555, 1992.

28 MINIM, V. P. R. Análise sensorial: estudos com consumidores. Viçosa: Ed. UFV, 2006. 225 p.

29 MOSCATTO, J.A.; PRUDÊNCIO-FERREIRA, S.H.; HAULLY, M.C. Farinha de yacon e inulina como ingredientes na formulação de bolo de chocolate. Ciência e Tecnologia de Alimentos, v.24, n.4, p.634-640, 2004.

30 MOURA, F. A. et al. Biscoitos tipo "cookie" elaborados com diferentes frações de semente de abóbora (Curcubita maxima). Alimentos e Nutrição, v.21, p.579-585, 2010.

31 OLIVEIRA, A.M.M.M.; MARINHO, H.A. Development of panettone made of peach palm flour (Bactris gasipaes Kunth). Alimentos e Nutrição, Araraquara, v.21, n.4, p.595-605, out./dez. 2010.

32 OLIVEIRA, S.P.; REYES, F.G.R. Biscoito com alto teor de fibra de milho: preparo, caracterização química e tecnológica e teste de aceitabilidade. Ciência e Tecnologia de Alimentos, Campinas, v.10, n.2, p.273-286, abr./jun. 1990.

33 PEREZ, P.M.P.; GERMANI, R. Elaboração de biscoitos tipo salgado, com alto teor de fibra alimentar, utilizando farinha de berinjela (Solanum melongena, L.). Ciência e Tecnologia de Alimentos, v.27, n.1, p.186-192, 2007.

34 PESTANA, V.R.; ZAMBIAZI, R.C.; MENDONÇA, C.R.; BRUSCATTO, M.H.; LERMA-GARCIA, M.J.; RAMIS-RAMOS, G. Quality changes and tocopherols and $\gamma$-orizanol concentrations. Journal of American Oil Chemists' Society, v.85, p.1013-1019, 2008.

35 PIZZINATTO, A.; MAGNO, C.P.R.; CAMPAGNOLLI, D.M.F. Avaliação tecnológica de produtos derivados da farinha de trigo (pão, macarrão, biscoitos). Campinas: Centro de Tecnologia de Farinhas e Panificação, Instituto de Tecnologia de Alimentos, 1993. 54 p.

36 PUMAR, M. et al. Avaliação do efeito fisiológico da farinha de semente de abóbora (Cucurbita maxima L.) no trato intestinal de ratos. Ciência e Tecnologia de Alimentos, Campinas, v. 28, p. 7-13, 2008. Suplemento.

37 PUMAR, M.; DA SILVA, M.C.; MARTINS, F. T. Frações obtidas no pré-preparo da abóbora bahiana (Cucurbita moschata). In: SIMPÓSIO LATINO AMERICANO DE CIÊNCIA DE ALIMENTOS, 4., 2001, Campinas-SP, 2001. Anais... Campinas: UNICAMP, 2001. v.1.

38 ROSENTHAL, A. J. Relation between instrumental and sensory measures of food texture. In: ROSENTHAL, A. J. (Ed.). Food texture: measurement and perception. Gaithersburg: Aspen, 1999. p.1-17

39 SAURA-CALIXTO, F. Fibra dietética de manzana: hacia nuevos tipos de fibras de alta calidad. Alimentaria, n.242, p.5761, 1993.

40 SILVA, M.R. Caracterização química e nutricional da farinha de jatobá (Hymenaea stigonocarpa Mart.): desenvolvimento e otimização de produtos através de testes sensoriais afetivos. 1997. 154 f. Tese (Doutorado em Ciência da Nutrição) - Curso de Pós-graduação em Tecnologia de Alimentos, Universidade Estadual de Campinas, Campinas, 1997.

41 SILVEIRA, A.J. et al. Elaboração e análise sensorial de minipanetone integral. In: SEMANA DE TECNOLOGIA EM ALIMENTOS, 6., 2008, Ponta Grossa. Trabalhos... Ponta Grossa: Departamento de Coordenação de Alimentos, Universidade Tecnológica Federal do Paraná, 2008. p.1-6.

42 SOUZA, M.L. et al. Processamento de cookies de castanha-do-brasil. In: CONGRESSO BRASILEIRO DE CIÊNCIA E TECNOLOGIA DE ALIMENTOS, 17., 2000, Fortaleza. Livros de Resumo... Campinas: SBCTA, 2000. v. 3.

43 STATSOFT, Inc. Statistica (data analysis software system). Version 7. Disponível em: www.statsoft.com. Acesso em: 27 de outubro 2012

44 SZCZESNIAK, A.S. Texture is a sensory property. Food Quality and Preference, Barking, v.13, n.4, p.215-225, 2002. 
45 TEIXEIRA, E.; MEINERT, E.M.; BARBETTA, P.A. Análise sensorial de alimentos. Florianópolis: UFSC, 1987. p. 60.

46 YAMASHITA, K.; IKEDA, S.; OBAYASHI, M. Comparative effects of flaxseed and sesame seed on vitamin E and cholesterol levels in rats. Lipids, v.38, n.12, p.1249-55, 2003. 